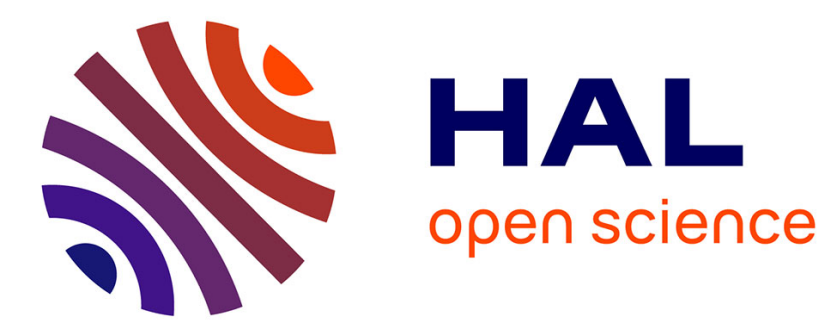

\title{
Targeted versus untargeted omics - the CAFSA story
}

Maria del Mar Amador, Benoit Colsch, Foudil Lamari, Claude Jardel, Farid Ichou, Agnès Rastetter, Frédéric Sedel, Fabien Jourdan, Clément Frainay, Ronald A Wevers, et al.

\section{- To cite this version:}

Maria del Mar Amador, Benoit Colsch, Foudil Lamari, Claude Jardel, Farid Ichou, et al.. Targeted versus untargeted omics - the CAFSA story. Journal of Inherited Metabolic Disease, 2018, 41 (3), pp.1-10. 10.1007/s10545-017-0134-3 . hal-01777969

\section{HAL Id: hal-01777969 \\ https://hal.sorbonne-universite.fr/hal-01777969}

Submitted on 25 Apr 2018

HAL is a multi-disciplinary open access archive for the deposit and dissemination of scientific research documents, whether they are published or not. The documents may come from teaching and research institutions in France or abroad, or from public or private research centers.
L'archive ouverte pluridisciplinaire HAL, est destinée au dépôt et à la diffusion de documents scientifiques de niveau recherche, publiés ou non, émanant des établissements d'enseignement et de recherche français ou étrangers, des laboratoires publics ou privés. 


\title{
Targeted versus untargeted omics - the CAFSA story
}

\author{
Maria del Mar Amador ${ }^{1} \cdot$ Benoit Colsch ${ }^{2} \cdot$ Foudil Lamari $^{3,4}$ - Claude Jardel $^{3,4} \cdot$ Farid Ichou $^{5} \cdot$ Agnès Rastetter $^{6}$. \\ Frédéric Sedel ${ }^{7} \cdot$ Fabien Jourdan $^{8} \cdot$ Clément Frainay $^{8} \cdot$ Ronald A. Wevers $^{9} \cdot$ Emmanuel Roze $^{1,4,6} \cdot$ Christel Depienne $^{10}$. \\ Christophe Junot $^{2} \cdot$ Fanny Mochel $^{4,6,11,12}$
}

\begin{abstract}
Background In 2009, untargeted metabolomics led to the delineation of a new clinico-biological entity called cerebellar ataxia with elevated cerebrospinal free sialic acid, or CAFSA. In order to elucidate CAFSA, we applied sequentially targeted and untargeted omic approaches.

Methods and results First, we studied five of the six CAFSA patients initially described. Besides increased CSF free sialic acid concentrations, three patients presented with markedly decreased 5-methyltetrahydrofolate (5-MTHF) CSF concentrations. Exome sequencing identified a homozygous POLG mutation in two affected sisters, but failed to identify a causative gene in the three sporadic patients with high sialic acid but low 5-MTHF. Using targeted mass spectrometry, we confirmed that free sialic acid was increased in the CSF of a third known POLG-mutated patient. We then pursued pathophysiological analyses of CAFSA using mass spectrometry-based metabolomics on CSF from two sporadic CAFSA patients as well as 95 patients with an unexplained encephalopathy and 39 controls. This led to the identification of a common metabotype between the two initial CAFSA patients and three additional patients, including one patient with Kearns-Sayre syndrome. Metabolites of the CSF metabotype were positioned in a reconstruction of the human metabolic network, which highlighted the proximity of the metabotype with acetyl-CoA and carnitine, two key metabolites regulating mitochondrial energy homeostasis.

Conclusion Our genetic and metabolomics analyses suggest that CAFSA is a heterogeneous entity related to mitochondrial DNA alterations either through $P O L G$ mutations or a mechanism similar to what is observed in Kearns-Sayre syndrome.
\end{abstract}

Fanny Mochel

fanny.mochel@upmc.fr

1 Assistance Publique-Hôpitaux de Paris, Département de Neurologie, La Pitié-Salpêtrière University Hospital, Paris, France

2 Service de Pharmacologie et Immuno-Analyse (SPI), Laboratoire d'Etude du Métabolisme des Médicaments, CEA, INRA, Université Paris Saclay, MetaboHUB, F-91191 Gif-sur-Yvette, France

3 Assistance Publique-Hôpitaux de Paris, Laboratoire de Biochimie Métabolique, La Pitié-Salpêtrière University Hospital, Paris, France

4 Université Pierre et Marie Curie, Groupe de Recherche Clinique Neurométabolique et Centre de Référence Neurométabolique Adulte, Paris, France

5 Institute of Cardiometabolism And Nutrition, ICAN, Metabolomics Core Facility, Paris, France
6 Sorbonne Universités, UPMC-Paris 6, UMR S 1127, and Inserm U 1127, and CNRS UMR 7225, and ICM, F-75013 Paris, France

7 MedDay Pharmaceuticals, Paris, France

8 Université de Toulouse, INRA, Université de Toulouse 3 Paul Sabatier, Toulouse, France

9 Radboud University Medical Centre, Translational Metabolic Laboratory, Department Laboratory Medicine, Nijmegen, the Netherlands

10 Hôpitaux Universitaires de Strasbourg, Unité de cytogénétique chromosomique et moléculaire, Strasbourg, France

11 Assistance Publique-Hôpitaux de Paris, Département de Génétique, La Pitié-Salpêtrière University Hospital, Paris, France

12 Reference Center for Neurometabolic Diseases, Department of Genetics, La Pitié-Salpêtrière University Hospital, 47 Boulevard de l’Hôpital, 75013 Paris, France 


\section{Introduction}

In 2009, an untargeted metabolomics approach using nuclear magnetic resonance spectroscopy (NMRS) led to the delineation of a new clinico-biological entity in six patients among a cohort of 144 patients presenting with a progressive and complex unknown neurological disease (Mochel et al 2009). The main clinical features of these patients were cerebellar ataxia, peripheral neuropathy and cognitive/psychiatric manifestations (Mochel et al 2009). While there was no overt sign of mitochondrial dysfunction on patient's muscle biopsy, their clinical presentation led to the clinical suspicion of a mitochondrial disease but several mitochondrial investigations were negative. NMRS showed a striking elevation of free sialic acid in the cerebrospinal fluid (CSF) of all six patients (Mochel et al 2009). The term cerebellar ataxia with elevated cerebrospinal free sialic acid (CAFSA) was proposed. Given the association of free sialic acid elevation in CSF with hyposialylation of transferrin, pathophysiological investigations were initially oriented toward sialic acid metabolism. Four candidate genes were investigated but did not bear any mutation in the CAFSA patients (Mochel et al 2009). Thus, we decided to take advantage of new genetic and metabolic tools to elucidate CAFSA. We applied sequentially targeted and untargeted omic approaches, including whole exome sequencing and mass spectrometry (MS)-based metabolomics, on a series of patients with cerebellar ataxia and elevated cerebrospinal free sialic acid.

\section{Patients and methods}

\section{Patients}

All patients described in this study were addressed to rare diseases reference centers (the Pitié-Salpêtrière university hospital, Paris, France mainly and the Radboud University Nijmegen Medical Center, The Netherlands) for diagnostic and therapeutic assessment. They were over 18 years and signed a written informed consent before participating in the studies (RCB 2010-A01395-34).

In order to elucidate the molecular basis of CAFSA, after our failed candidate gene approach (Mochel et al 2009), we decided to first conduct an untargeted genetic analysis using whole exome sequencing. Out of the six CAFSA patients initially described, DNA was available in sufficient amount for five of them. Unfortunately, none of the parents' DNA was accessible so we were not able to perform trio analyses. For the patients whose exome sequencing was inconclusive
(3/5), we wished to pursue pathophysiological analyses and understand their peculiar CSF profile using MS-based metabolomics. CSF was available for two out of three patients and were included in a larger CSF study performed in 134 patients selected from a CSF biobank collected from adult patients seen in the neurometabolic research group of the PitiéSalpetrière university hospital and previously described (Mochel et al 2009). Briefly, 95 patients presented with an unexplained encephalopathy, i.e., patients with progressive neurological disorders that were selected based on clinical and radiological features suggestive of an inborn error of metabolism. Additionally, we included 39 negative controls, i.e., subjects without progressive neurological disease, for whom a lumbar puncture (LP) had been performed because of headache, dizziness, non-organic psychogenic neurologic symptoms, non specific pain or spinal disc herniation. In all cases, the presence of an underlying neurological disease was discarded by thorough diagnostic work-up. LP were performed for a routine work-up or follow-up of all patients and not solely for research purposes.

\section{Measurement of sialic acid by MS}

Sialic acid in CSF was measured by UPLC-MS/MS according to the method reported for sialic acid determination in urine (Van der Ham et al 2007), with modifications as detailed in eMethod in the Supplementary material. Intra- and inter-assay variation in CSF was determined by analyzing a mix of ten CSF obtained from controls (see above) and was 3.34\% and $6.13 \%$, respectively. Limit of detection was $1.15 \mu \mathrm{M}$ and limit of quantification was $3.12 \mu \mathrm{M}$. Free sialic acid calibration curve was linear between 3.12 and $200 \mu \mathrm{M}$ with $\mathrm{R}^{2}=0.998$, as obtained by linear regression. The concentration of free sialic acid determined in the CSF from 39 controls (see above) was $11 \pm 4.8 \mu \mathrm{M}$, which is very similar to the concentration reported in controls by NMRS (Mochel et al 2009).

\section{Whole exome sequencing}

Genomic DNA of patients was extracted from blood cells using standard phenol-chloroform procedures. Exome sequencing was performed by DNAvision (Charleroi, Belgium). Genomic DNA was captured using the SureSelect Human All Exon $50 \mathrm{Mb}$ kit (Agilent), followed by 75 base pair paired-end massively parallel sequencing on a HiSeq2000 sequencing system (Illumina), according to the manufacturers' instruction and protocols. Reads were mapped onto the reference genome (hg19) using the Burrows-Wheeler Aligner software (Li et al 2009; Li and Durbin 2010). Removing of duplicated reads and mutation 
calling were performed using samtools ( $\mathrm{Li}$ et al 2009; $\mathrm{Li}$ and Durbin 2010) according to the following criteria: position coverage greater or equal to 20 , mutation proportion greater or equal to $25 \%$, and mutated bases called at least $10 \%$ on each strand. Filtering of variants included i) a minor allele frequency $(\mathrm{MAF}) \leq 1 \%$ in Hapmap, 1000 Genomes, and Exome variant server, ii) a predicted deleterious impact on the gene/protein (indels introducing frameshifts, in-frame insertions or deletions, nonsense or splice-site mutations, mutations altering start or termination codons, or non-synonymous variants predicted to be possibly deleterious by SIFT or Polyphen-2), and iii) a variant status compatible with an autosomal recessive inheritance (homozygous or compound heterozygous variants). Mutation interpretation was assessed using Alamut2.1 (Interactive Biosoftware).

\section{MS-based metabolomics}

Samples, chemicals and reagents, CSF metabolite extraction, experimental settings for liquid chromatography coupled to high-resolution mass spectrometry (LC-HRMS), data processing, metabolite identification criteria, and visualization of metabotypes are detailed in the eMethod in the Supplementary material. CSF samples were centrifuged, decanted, and immediately frozen at $-80^{\circ} \mathrm{C}$ after LP. Sample analysis was performed in three independent experiments (batches). Quality control samples were injected at regular intervals throughout the experiments. They aimed at correcting analytical drifts within each batch and performing batch fusion.

CSF samples were treated by methanol for protein precipitation. LC-HRMS analyses were performed using a NEXERAShimadzu liquid chromatography system (Champs-sur-Marne, France) coupled to an Exactive mass spectrometer from Thermo Fisher Scientific (Courtaboeuf, France) fitted with an electrospray source operated in the positive and negative ion modes. The ultra-high performance liquid chromatographic (UHPLC) separation was performed on a Hypersil GOLD C8 $1.9 \mu \mathrm{m}, 2.1 \mathrm{~mm} \times 150 \mathrm{~mm}$ column at $30{ }^{\circ} \mathrm{C}$ (Thermo Fisher Scientific, les Ulis, France). Mobile phases were $100 \%$ water in $\mathrm{A}$ and $100 \% \mathrm{ACN}$ in $\mathrm{B}$, both containing $0.1 \%$ formic acid.

Regarding data processing, all raw data were manually inspected using the Qualbrowser module of Xcalibur version 2.1 (Thermo Fisher Scientific, Courtaboeuf, France), and automatic peak detection and integration were performed using the XCMS software package (Smith et al 2006). XCMS returned two data matrices (i.e., corresponding to detection and chromatography conditions) containing $\mathrm{m} / \mathrm{z}$ and retention time values of features together with their concentrations expressed in arbitrary units (i.e., areas of chromatographic peaks). Features were annotated by matching their accurate measured masses $\pm 10 \mathrm{ppm}$ with theoretical masses contained in biochemical and metabolomic databases by using an informatics tool developed in $\mathrm{R}$ language. They were also annotated by our spectral database according to accurate measured masses and chromatographic retention times (Roux et al 2012; Boudah et al 2014; Weiss et al 2016). Features corresponding to identified or putatively annotated ions were selected. Data were expressed as ratios of individual levels observed for patients and controls to control mean levels. A metabolite was considered to be present in a sample at an abnormal concentration as soon as it was above the [mean concentration $+2 \mathrm{SD}$ ] value (SD: standard deviation of metabolite concentrations in a given experiment).

Network of metabotype analysis Recon 2 version 3 human genome scale metabolic network was used as a context for metabotype analysis (Thiele et al 2013). Recon2 aims at gathering all metabolic reactions which can occur in human cells or tissues (7440 reactions and 2626 metabolites) in a single mathematical framework called a network (more formally a graph). This large knowledge resource allows going further than just focusing on few key metabolic pathways. It is of particular interest for holistic approaches. Such as untargeted metabolomics. Which may reveal biochemical modulations spanning several metabolic pathways. Recon 2 contains information on cellular localization of metabolites which cannot be obtained from metabolomics analysis. Hence, the network was modified to obviate compartment segmentation. The network was turned into a compound graph where nodes represent metabolites. Two nodes are connected by edges if the two corresponding metabolites are substrates and products of the same reaction. In order to keep only relevant substrate to product pairs, the graph was filtered by keeping only pairs of substrate product exchanging at least one carbon (atom mapping was computed using the Reaction Decoder Toolkit (Rahman et al 2016). Inorganic carbonated compounds such as $\mathrm{CO}_{2}$ were also removed. Shortest path distance matrix was computed between each pair of metabolites in the metabotype. The matrix was represented as a heatmap using R HeatMap2 package, grouping rows and columns according to hierarchical clustering method with complete linkage for aggregation and Euclidean distance. Sub-networks characterizing each cluster were created from the union of all shortest paths between cluster members. These sub-networks were displayed using MetExplore web server (Cottret et al 2010).

\section{Results}

\section{Patient characteristics and targeted analyses}

Table 1 (upper section) summarizes the main clinical and biochemical characteristics of five out of the six CAFSA patients previously reported (Mochel et al 2009). CSF free sialic acid concentrations from the five patients ranged from 36 to $67 \mu \mathrm{M}$. Clinically, cerebellar ataxia manifested in adulthood 


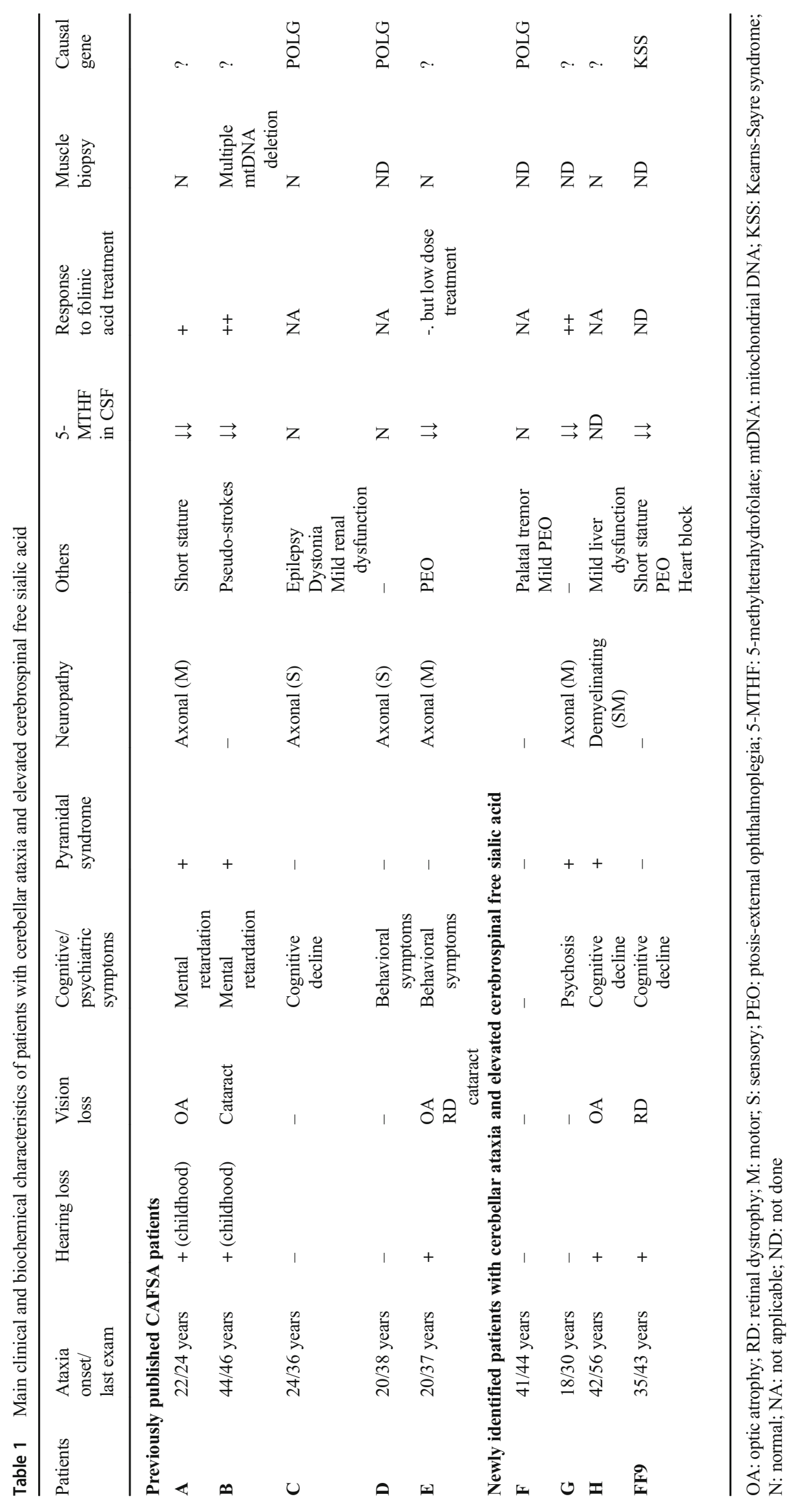


(20-44 years), and was associated with peripheral neuropathy, audition and/or vision loss, and cognitive/psychiatric symptoms. The additional occurrence of pseudo-strokes, ptosisophthalmoplegia and renal dysfunction in some patients was even more suggestive of a mitochondrial dysfunction. Nonetheless, muscle histopathology was normal and only one patient (patient B) displayed multiple mitochondrial DNA deletions on muscle biopsy. Mitochondrial DNA point mutations were excluded in all patients as well as POLG mutations in patient B. Besides sialic acid, we measured 5methyltetrahydrofolate (5-MTHF) concentrations in patient's CSF (Perry et al 2009) and found a striking decrease in three of them. Importantly, two patients were markedly improved with folinic acid supplementation in terms of gait and ambulation, while the third patient did not show overt benefit, but only received low dose folinic acid.

\section{Whole exome sequencing}

Exome sequencing was undertaken in the three sporadic patients (subjects A, B, E) and two affected sisters (subjects C and D) under the assumption that these patients had a disorder with autosomal recessive inheritance. Filtering of rare, possibly deleterious variants in the homozygous state in both sisters revealed variants in 11 different genes, including a known pathogenic mutation in POLG (NM_002693.2:c.2243G > C, p.(Trp748Ser)), which was further confirmed by Sanger sequencing in both individuals. There was no gene with at least two heterozygous variants common to both sisters. None of these three sporadic patients had variants in $P O L G$ - which we previously excluded in patient B - or in genes involved in mitochondrial DNA maintenance such as Twinkle.

For the three sporadic patients (A, B, and E), special attention was given to genes encoding for proteins involved in sialic acid or folate metabolism, but that was inconclusive. Filtering of rare, possibly deleterious homozygous variants in individual A, who had consanguineous parents revealed 31 variants in 29 genes and ten variants on chromosome X. Analysis of individual $B$ and $E$ identified 15 and 24 in 11 and 18 autosomal genes, respectively, and five and six genes with at least two variants. Venn diagram showed six genes with variants in all three individuals: FAM153B, RGPD5, RGPD8, PRAMEF11, OR11H1, $O R 11 H 2$ (eFigure in the Supplementary material). However, all variants proved to be polymorphisms, with frequency below our threshold but relatively frequent in control populations (ExAC database), in genes tolerant to variations. Hence, this approach did not permit identification of the causative variants in the sporadic cases, suggesting that CAFSA is genetically heterogeneous.

Since two out the five CAFSA patients ended up bearing mutations in $P O L G$, we wanted to assess whether free sialic acid was elevated in other patients with known mutations in POLG. We were able to retrieve the CSF of one such patient and found a significant elevation of free sialic acid in her CSF $(38 \mu \mathrm{M})$. This patient's clinical presentation is summarized in Table 1 (lower panel, patient F).

\section{MS-based metabolomics}

Detection of CSF metabolites The LC-HRMS based analysis of CSF metabolome was previously performed in our laboratory by combining reverse phase and hydrophilic interaction liquid chromatography. This led to the identification of 122 metabolites (Weiss et al 2016). Here, metabolite detection was achieved using reverse phase chromatography, and 89 metabolites, whose analytical information and identification status are displayed in eTable 1 in the Supplementary material, were selected from data processing steps (see eMethod in the Supplementary material). The detected CSF metabolome was composed of $37 \%$ amino acids and derivatives. Other detected species were peptides, acylcarnitines, carboxylic acids and derivatives, and nucleosides and nucleotidic bases.

The CSF metabotype of CAFSA patients and identification of additional patients with cerebellar ataxia and elevated cerebrospinal free sialic acid The CSF metabotype of the two CAFSA patients (A and $\mathrm{B}$ ) revealed that, in addition to increased sialic acid concentrations by a factor of 4 to 5 , they also exhibited many other abnormally elevated metabolite concentrations, including sugar derivatives, such as acetylhexosamine (putatively acetyl-glucosamine, acetylgalactosamine or acetyl-mannosamine), deoxyribose, xylonic acids, and isomers, threonic/erythronic acids, and adonitol/xylitol, acylcarnitines, acetylated compounds (many amino acids) and other metabolites, such as N-ribosylhistidine and succinyladenosine (Table 2 and eTable 3 in the Supplementary material), the latter being known to accumulate in patients with adenylosuccinate lyase deficiency. In addition, among the 134 individuals analyzed by MS-based metabolomics, this metabotype was very similar to that of two other patients $(G$ and $\mathrm{H}$ ), including increased free sialic acid concentrations. In patient $\mathrm{G}$, targeted MS analysis confirmed very high levels of free sialic acid $(78 \mu \mathrm{M})$. The clinical characteristics of patients $\mathrm{G}$ and $\mathrm{H}$ are reported in Table 1 (lower panel). Besides cerebellar ataxia, these two patients also presented with peripheral neuropathy and cognitive/psychiatric symptoms. Patient $\mathrm{H}$ manifested with vision loss due to optic atrophy and mild liver dysfunction, evocative of a mitochondrial dysfunction, but his muscle biopsy and mitochondrial DNA analyses were normal. Exome sequencing had also been performed in patient $\mathrm{H}$, as part of a research project on patients with a suspected mitochondrial dysfunction (Fondation Maladies Rares, principal investigator: Claude Jardel), but failed to identify any causal mutation. Furthermore, 5-MTHF concentration was measured in the CSF of patient G and was extremely low. Similar to 
patients $\mathrm{A}$ and $\mathrm{B}$, the motor functions of patient $\mathrm{G}$ responded very well to folinic acid supplementation.

Interestingly, hierarchical clustering analyses performed on the 95 patients with unexplained encephalopathies plus CAFSA patients A and B showed that patients B and G clustered with patient FF9, who was diagnosed with Kearns-Sayre syndrome (Fig. 1). Targeted MS analysis confirmed that free sialic acid was significantly elevated in this patient with Kearns-Sayre syndrome $(60 \mu \mathrm{M})$. In addition, patients A and $\mathrm{H}$ were grouped in another cluster. Metabolic abnormalities were more pronounced in patients B, G, and FF9 (Table 2), than in patients $\mathrm{A}$ and $\mathrm{H}$ who mainly exhibited increased concentrations of sugar derivatives and acylcarnitines (Table 2).

Mapping of the CAFSA metabotype in the context of human genome scale metabolic network CSF metabotype is not necessarily the outcome of the modulation of a single metabolic pathway. Several, potentially disconnected, metabolic processes may be leading to metabolite concentration changes in the CSF. To test this hypothesis, metabolites of the CSF metabotype were then positioned in a reconstruction of the human metabolic network (Recon2), containing thousands of metabolites and reactions (Thiele et al 2013), in order to know if they were located in the same "area" of the network; thus, taking place in the same metabolic processes, and also if they could be closely linked to sialic acid and/or folic acid metabolism. Forty-one out of the 89 metabolites considered for this study were found in the Recon 2 network (eTable 2 in the Supplementary material). Folic acid, which was not detected in our experimental conditions, was added to the list to decipher its connection to the rest of the metabotype.
To formally evaluate closeness in terms of metabolism between the elements of the metabotype, a distance analysis was first performed. Two metabolites were considered close if few biochemical reactions were required to transform one into the other, and far away if it required several reactions (Frainay and Jourdan 2017). Accordingly, a distance matrix was built where rows and columns were classified using hierarchical clustering and color of cells corresponded to short distances (red) to long distances (light yellow) between two metabolites (Fig. 2). Likewise, we observed a core cluster (black) containing mainly sub-clusters of carnitines and acetylated compounds and citrate. The sub-network corresponding to this black cluster (Fig. 3) highlighted the proximity of the metabotype with acetyl-CoA and carnitine, two key metabolites regulating mitochondrial energy homeostasis. A peripheral cluster (blue) containing sub-clusters of aromatic aminoacids, nucleosides, and puric bases was also observed (Fig. 2). Folic acid was far from most other detected CSF metabolites, and sialic acid was only connected to acetyl-hexosamine (i.e., putatively acetyl-glucosamine, acetyl-galactosamine or acetyl-mannosamine) (Fig. 3).

\section{Discussion}

Using a combination of targeted and untargeted genetic and metabolomics analyses, we were able to further delineate the underlying causes of CAFSA. Two patients harbored mutations in $P O L G$ and we showed in the CSF of a third POLGmutated patient that free sialic acid was indeed elevated. In addition, MS-based metabolomics led to the identification of three additional patients with cerebellar ataxia and elevated

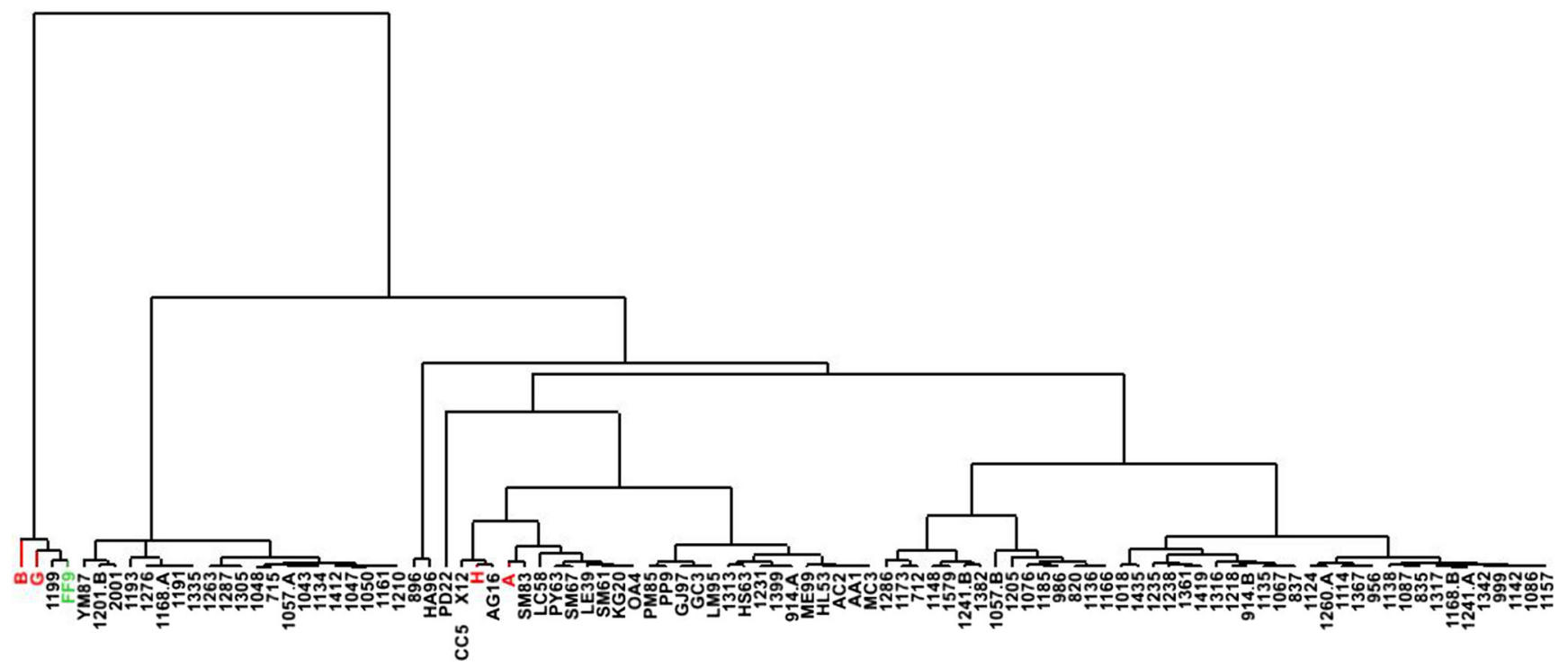

Fig. 1 Hierarchical clustering analyses performed on patients with CAFSA and patients with unexplained encephalopathies. Eighty-nine metabolites were taken into account. Chromatographic peak areas of these metabolites were first mean-centered and scaled to unit variance before performing hierarchical clustering analysis using SIMCA-P12 software (Umetrics, Umea, Sweden) 
Table 2 CSF metabotype from four CAFSA patients and one patient with Kearns-Sayre syndrome, belonging to the same clusters

\begin{tabular}{|c|c|c|c|c|c|c|}
\hline & Patient ID & $\mathbf{G}^{\mathbf{a}}$ & $\mathrm{B}^{\mathrm{a}}$ & $\mathbf{A}^{\mathbf{b}}$ & $\mathbf{H}^{\mathbf{b}}$ & FF9 $^{b}$ \\
\hline Name & Chemical taxonomy $^{c}$ & CAFSA & CAFSA & CAFSA & CAFSA & KSS \\
\hline Carnitine & Carnitines & 1.94 & 1.03 & 1.61 & 1.08 & 0.71 \\
\hline Acetylcarnitine & $\mathrm{NC}$ & 2.04 & 1.37 & 1.74 & 1.66 & 1.59 \\
\hline Propionylcarnitine & Acylcarnitines & 1.75 & 1.00 & 1.33 & 1.67 & 1.15 \\
\hline $\begin{array}{l}\text { Butyrylcarnitine / } \\
\text { Isobutyrylcarnitine }\end{array}$ & Acylcarnitines & 1.79 & 0.94 & 1.46 & 1.38 & 4.66 \\
\hline $\begin{array}{l}\text { 2-ethylacrylylcarnitine / } \\
\text { Tiglylcarnitine }\end{array}$ & Acylcarnitines & 1.61 & 2.69 & 0.93 & 1.85 & 3.24 \\
\hline $\begin{array}{l}\text { 2-methylbutyroyl-/ valeryl- } \\
\text { /isovalerylcarnitine }\end{array}$ & Acylcarnitines & 1.59 & 1.05 & 2.15 & 1.40 & 2.79 \\
\hline 3-hydroxyisovalerylcarnitine & Acylcarnitines & 1.29 & 1.27 & 0.67 & 1.16 & 1.69 \\
\hline 3-methyl-2-oxovaleric acid & Keto-acids \& derivatives & 1.56 & 0.68 & 0.41 & 1.24 & 1.60 \\
\hline $\begin{array}{l}\text { 4-methyl-2-oxovaleric acid } \\
\text { (ketoleucine) }\end{array}$ & Keto-acids \& derivatives & 1.86 & 0.50 & 0.37 & 0.94 & 1.59 \\
\hline Stachydrine (proline-betaine) & Carb. acids \& derivatives & 3.68 & 0.14 & 2.61 & 2.93 & 0.02 \\
\hline Malic acid & Dicarb. acids \& derivatives & 0.64 & 1.25 & 0.67 & 1.32 & 1.13 \\
\hline Cis-aconitic acid & Tricarb. acids \& derivatives & 1.82 & 1.93 & 1.74 & 2.05 & 1.09 \\
\hline Citric acid & Tricarb. acids \& derivatives & 1.46 & 0.93 & 1.12 & 1.37 & 1.17 \\
\hline $\begin{array}{l}\text { N2-succinyl-glutamic acid 5- } \\
\text { semialdehyde }\end{array}$ & N-acyl-alpha-amino acids & 2.18 & 2.63 & 1.35 & 1.80 & 2.08 \\
\hline Acetyl-glycine & N-acyl-alpha-amino acids & 0.55 & 0.85 & 0.33 & 0.37 & 0.95 \\
\hline Acetyl-alanine & N-acyl-alpha-amino acids & 0.78 & 1.63 & 0.22 & 1.31 & 1.33 \\
\hline Acetyl-serine & N-acyl-alpha-amino acids & 1.63 & 3.18 & 0.61 & 1.32 & 2.22 \\
\hline $\begin{array}{l}\text { Acetyl-valine or acetyl- } \\
\text { norvaline }\end{array}$ & N-acyl-alpha-amino acids & 0.96 & 1.45 & 0.38 & 1.04 & 1.56 \\
\hline N-acetyl-methionine & N-acyl-alpha-amino acids & 0.52 & 5.49 & 0.00 & 0.77 & 2.09 \\
\hline N-acetyl-phenylalanine & N-acyl-alpha-amino acids & 0.23 & 0.69 & 0.33 & 0.28 & 0.66 \\
\hline Phenylacetyl-glutamine & N-acyl-alpha-amino acids & 0.49 & 1.00 & 0.27 & 1.03 & 0.57 \\
\hline Leucyl-alanine & Dipeptides & 4.45 & 3.79 & 0.96 & 1.43 & 1.53 \\
\hline Prolyl-hydroxyproline & Dipeptides & 0.85 & 1.15 & 0.53 & 0.74 & 1.00 \\
\hline Leucyl-proline & Dipeptides & 3.12 & 3.64 & 1.64 & 1.29 & 3.25 \\
\hline Leucyl-aspartic acid & Dipeptides & 1.39 & 1.66 & 0.71 & 1.35 & 1.50 \\
\hline Acetyl-aspartylglutamic acid & Dipeptides & 2.14 & 2.75 & 0.86 & 0.48 & 1.01 \\
\hline Quinic acid & $\mathrm{NC}$ & 0.88 & 4.97 & 0.05 & 0.94 & 0.66 \\
\hline Dehydroquinic acid & Cyclitols \& derivatives & 3.93 & 3.01 & 2.08 & 1.41 & 1.82 \\
\hline Anhydro-sorbitol & Monosaccharides & 1.26 & 0.69 & 1.38 & 0.32 & 0.59 \\
\hline Acetyl-hexosamine* & Acylaminosugars & 2.33 & 2.62 & 2.36 & 1.63 & 2.29 \\
\hline Deoxyribose & Pentoses & 4.18 & 8.74 & 1.95 & 1.97 & 7.89 \\
\hline Methylthioribose & Pentoses & 0.55 & 2.24 & 0.22 & 0.57 & 0.95 \\
\hline Xylonic acid or isomers & Sugar acids & 3.74 & 4.17 & 2.82 & 1.78 & 4.12 \\
\hline Threonic acid /Erythronic acid & Sugar acids \& derivatives & 2.48 & 2.14 & 2.32 & 1.48 & 1.71 \\
\hline Glyceric acid & Sugar acids \& derivatives & 1.24 & 1.23 & 0.80 & 1.87 & 1.20 \\
\hline Adonitol /Xylitol & Sugar alcohols & 3.34 & 4.27 & 2.57 & 1.37 & 3.14 \\
\hline Mannitol & Sugar alcohols & 2.20 & 2.16 & 0.95 & 1.05 & 1.61 \\
\hline $\mathrm{N}$-acetylneuraminic acid & $\mathrm{N}$-acylneuraminic acids & 5.19 & 5.06 & 3.96 & 1.50 & 3.89 \\
\hline
\end{tabular}

KSS: Kearns-Sayre syndrome; UK: unknown; Carb.: carboxylic; a: mean values from three experiments; b: Values from one experiment; c: From HMDB; NC: Not classified; yellow: value > mean + 1SD; orange: value > mean +2SD; red: value > mean + 3SD; *putatively acetyl-glucosamine, acetyl-galactosamine, or acetyl-mannosamine 


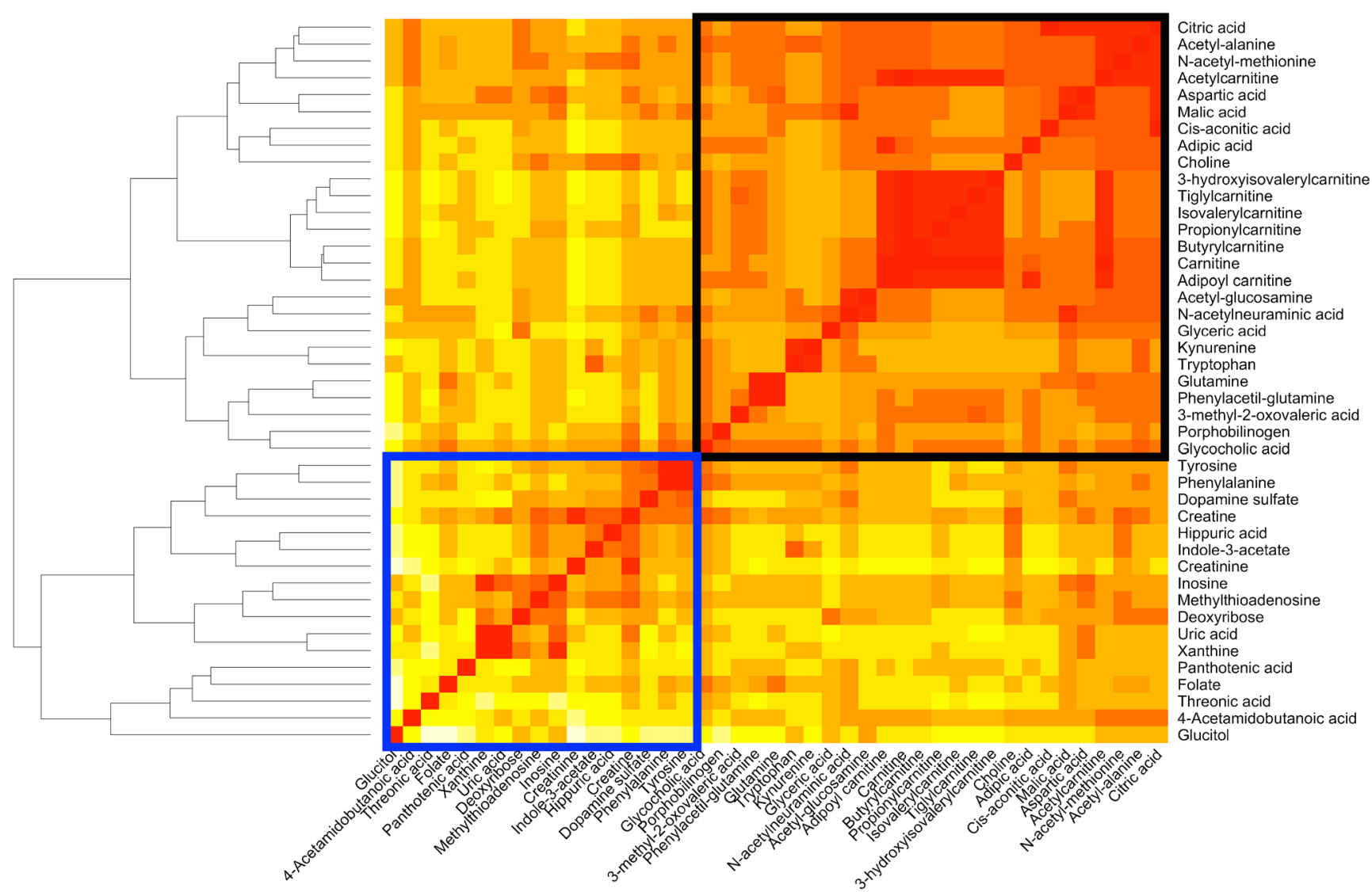

Fig. 2 Distance matrix on the entire fingerprint. Each cell of the matrix is colored according to distance between the two corresponding entries, from red (distance $=0$ to white distance $=9$ ). We observed a core cluster

(black) containing mainly sub-clusters of carnitines and acetylated compounds and citrate and a peripheral cluster (blue) containing subclusters of aromatic amino-acids, nucleosides, and puric bases

cerebrospinal free sialic acid, including one patient with Kearns-Sayre syndrome. MS-based metabolomics also showed the implication of additional metabolites related to mitochondrial energy homeostasis, specifically acetylCoA and carnitine. Furthermore, 5-MTHF was extremely low in the CSF of the three initial CAFSA patients that were tested as well as in two novel patients with cerebellar ataxia and elevated cerebrospinal free sialic acid, including the patient with Kearns-Sayre syndrome as previously described (Quijada-Fraile et al 2014), but was normal in $P O L G$-mutated patients. Although we did not evidence any mutation in other genes involved in mitochondrial DNA maintenance than POLG, one patient displayed multiple mitochondrial DNA deletions. Altogether, our genetic and metabolomics analyses suggest that CAFSA is related to mitochondrial DNA alterations either through $P O L G$ mutations or a mechanism similar to what is observed in Kearns-Sayre syndrome.

Back in 2009, we suspected that CAFSA was genetically heterogeneous, which we confirmed in the present study. Although our exome analysis was driven by metabolic hypotheses on sialic acid, folate, and mitochondrial metabolism, it was only successful in two siblings out of six patients, which emphasizes the complexity of next generation sequencing when parents' DNA is not available. Still, targeted and untargeted metabolic approaches using MS allowed us to expand our characterization of CAFSA taking advantage of the increased sensitivity of MS technology compared to NMRS. First, hierarchical clustering analysis performed on metabolomic data showed that non POLG-mutated CAFSA patients belonged to two distinct clusters (Fig. 1), emphasizing the heterogeneity of this entity. Main concentration variations observed in the CAFSA metabotype were related to sugar derivatives, acylcarnitines, carboxylic acid derivatives, acetylated amino acids, and dipeptides. Sugar derivatives are of special interest since their concentrations were dramatically modified in the two clusters. Notably, acetyl-hexosamines (i.e., putatively acetyl-glucosamine, acetyl-galactosamine or acetyl-mannosamine), whose levels were increased in CAFSA patients, are direct precursors of acetyl-neuraminic acid, also called sialic acid. Such findings would need to be confirmed by an alternative analytical method enabling the discrimination 


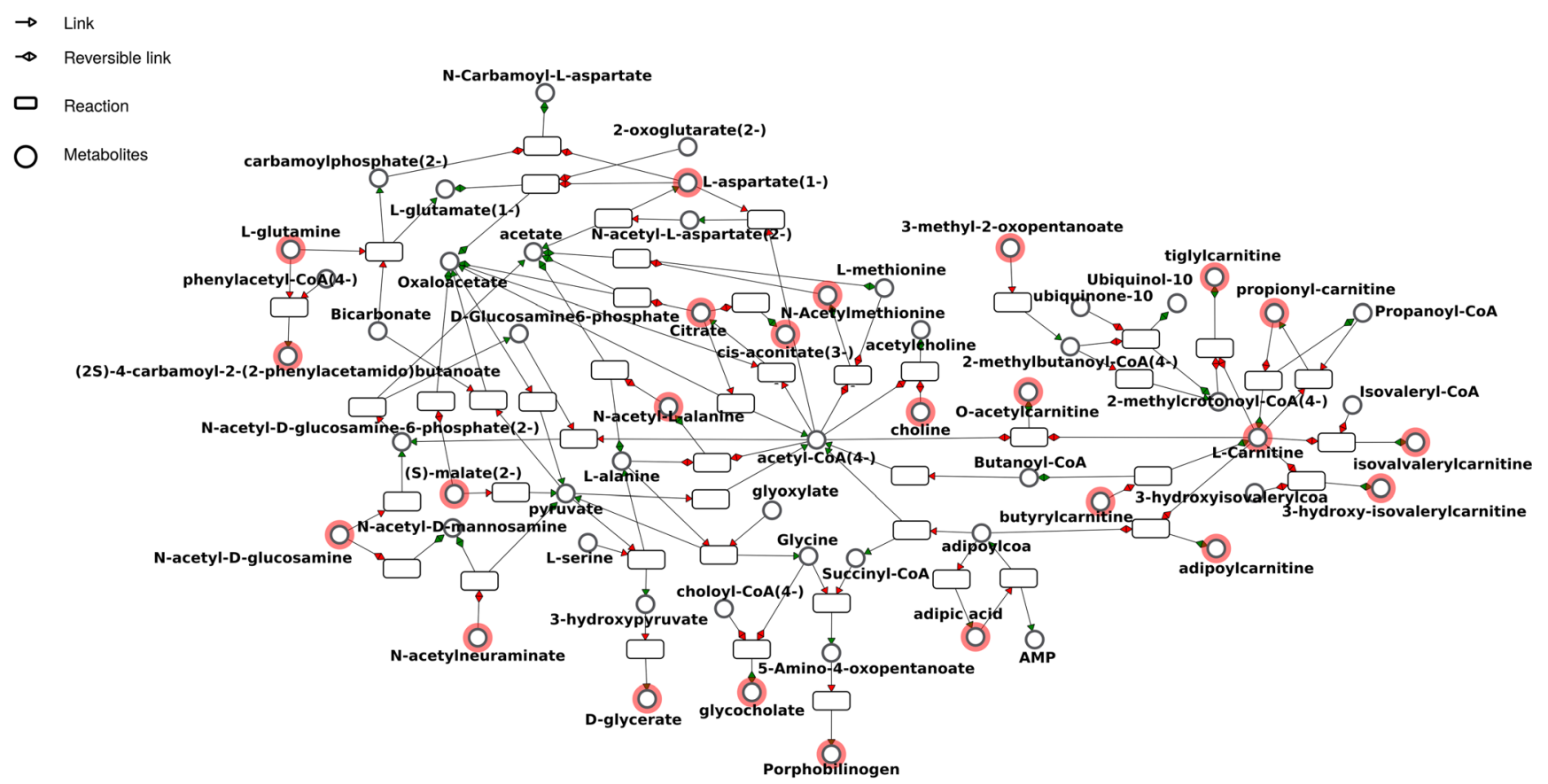

Fig. 3 Sub-network corresponding to the black cluster built from the distance matrix by keeping only the shortest paths between metabolites belonging to the cluster, and displayed using the MetExplore web server (see the Patients and methods section for additional information). Red circled nodes are the ones belonging to fingerprint. The nomenclature used for metabolite identification, which mentions the enantiomer information, is that of Recon2 between isomers. Second, we identified 5-MTHF deficiency as an additional CSF biomarker in these patients with high free sialic acid but no POLG mutations. Third, in non $P O L G$-mutated patients, we discovered a larger common metabotype pointing to alterations of mitochondrial energy homeostasis and resembling that of a patient with Kearns-Sayre syndrome. Therefore, even if our efforts were only partially successful at elucidating CAFSA, we advocate the combination of next generation sequencing with advanced metabolomics to expand our understanding of complex neurodevelopmental and/or neurodegenerative entities. Recently, such an approach elegantly proved to also be successful in identifying novel inborn errors of metabolism (Tarailo-Graovac et al 2016).

Mitochondrial diseases remain the largest and most heterogeneous entities among inborn errors of metabolism due to the large number of nuclear genes encoding mitochondrial proteins, as well as the additional contribution of the mitochondrial DNA. Lactate is a well-known biomarker of mitochondrial dysfunction, and others have been suggested recently, such as creatine, FGF21, and GDH15. 5-MTHF deficiency has been described mainly in patients with Kearns-Sayre syndrome (Quijada-Fraile et al 2014) and recent studies showed that mitochondrial DNA replication defects remodel one-carbon metabolism, which involves folate, through altered dNTP pools
(Nikkanen et al 2016). As exemplified by our patients, 5-MTHF deficiency shall be looked for in the CSF of patients suspected of mitochondrial diseases since supplementation with folinic acid, in sufficient amount, may greatly improve their motor functions. Conversely, the elevation of free sialic acid in CAFSA patients remains perplexing as there is no clear link between sialic acid and mitochondrial metabolism. In our initial NMRS-based metabolomics study, sialic acid was measured in several patients with known mitochondrial defects but was normal (Mochel et al 2009). Therefore, increased CSF free sialic acid may point to a subset of mitochondrial diseases involving mitochondrial DNA. It also remains to be determined whether free sialic acid can be a reliable biomarker of $P O L G$ mutations and/or Kearns-Sayre syndrome.

Details of funding This study was supported by a grant from the French Ministry of Health (PHRC SPECTMET-II, ID RCB 2010-A01395-34). The authors confirm independence from the sponsors.

\section{Compliance with ethical standards}

Conflict of interest Maria del Mar Amador, Benoit Colsch, Foudil Lamari, Claude Jardel, Farid Ichou, Agnès Rastetter, Emmanuel Roze, Ronald Wevers, Christel Depienne, Christophe Junot, and Fanny Mochel declare that they have no conflict of interest.

Frédéric Sedel is the CEO of MedDay Pharmaceuticals. 
Animal rights This article does not contain any studies with animal subjects performed by the any of the authors.

\section{References}

Boudah S, Olivier M-F, Aros-Calt S et al (2014) Annotation of the human serum metabolome by coupling three liquid chromatography methods to high-resolution mass spectrometry. J Chromatogr B Analyt Technol Biomed Life Sci 966:34-47

Cottret L, Wildridge D, Vinson F et al (2010) MetExplore: a web server to link metabolomic experiments and genome-scale metabolic networks. Nucleic Acids Res 38:W132-W137

Frainay C, Jourdan F (2017) Computational methods to identify metabolic sub-networks based on metabolomic profiles. Brief Bioinform 18:43-56

Li H, Durbin R (2010) Fast and accurate long-read alignment with burrows-wheeler transform. Bioinformatics 26:589-595

Li H, Handsaker B, Wysoker A et al (2009) The sequence alignment/map format and SAMtools. Bioinformatics 25:2078-2079

Mochel F, Sedel F, Vanderver A et al (2009) Cerebellar ataxia with elevated cerebrospinal free sialic acid (CAFSA). Brain 132:801-809

Nikkanen J, Forsström S, Euro L et al (2016) Mitochondrial DNA replication defects disturb cellular dNTP pools and remodel one-carbon metabolism. Cell Metab 23:635-648

Perry M, Li Q, Kennedy RT (2009) Review of recent advances in analytical techniques for the determination of neurotransmitters. Anal Chim Acta 653:1-22
Quijada-Fraile P, O’Callaghan M, Martín-Hernández E et al (2014) Follow-up of folinic acid supplementation for patients with cerebral folate deficiency and Kearns-Sayre syndrome. Orphanet J Rare Dis 9:217-225

Rahman SA, Torrance G, Baldacci L et al (2016) Reaction decoder tool (RDT): extracting features from chemical reactions. Bioinformatics 32:2065-2066

Roux A, Xu Y, Heilier J-F et al (2012) Annotation of the human adult urinary metabolome and metabolite identification using ultra high performance liquid chromatography coupled to a linear quadrupole ion trap-orbitrap mass spectrometer. Anal Chem 84:6429-6437

Smith CA, Want EJ, O'Maille G et al (2006) XCMS: processing mass spectrometry data for metabolite profiling using nonlinear peak alignment, matching, and identification. Anal Chem 78:779-787

Tarailo-Graovac M, Shyr C, Ross CJ et al (2016) Exome sequencing and the management of neurometabolic disorders. N Engl J Med 374: 2246-2255

Thiele I, Swainston N, Fleming RM et al (2013) A community-driven global reconstruction of human metabolism. Nat Biotechnol 31: 419-425

van der Ham M, Prinsen BH, Huijmans JG et al (2007) Quantification of free and total sialic acid excretion by LC-MS/MS. J Chromatogr B Analyt Technol Biomed Life Sci 848:251-257

Weiss N, Saint Hilaire PB, Colsch B et al (2016) Cerebrospinal fluid metabolomics highlights dysregulation of energy metabolism in overt hepatic encephalopathy. J Hepatol 65:1120-1130 